\title{
Dark Energy and Cosmological Model
}

\author{
R. G. Deshmukh \\ Department Of Physics, Shri Shivaji Arts, Commerce And Science College, Akola, Maharashtra (India)
}

\begin{abstract}
Today the space of our universe is filled with invisible stuff matter which expanding under the action of gravity which was dark matter. In 1990's astronomical observation and theoretical calculation was leading astrophysicists to believe that not only the dark matter but also there is vacuum empty space filled in universe that is dark energy. It is suggested that the apparently disparate cosmological phenomenon attributed to so called "dark matter" and dark energy arise from quantum level of space -time itself. This creation of space time results in metric expansion. A recent modification of Einstein's theory of general relativity by Chadwick, Hodgkinson and McDonald incorporate space time expansion. Recent evidence predicts that apparent amount of dark matter increases with age of universe. In addition proposal leads to the same result for the small but nonvanishing cosmological constant, related to dark energy.
\end{abstract}

Key words: Dark Energy, Dark Matter, Universe, Astrophysics.

\section{Introduction}

One of the most unexpected revelations about our understanding of the universe is that the universe is not dominated by the ordinary baryonic matter, but instead, by a form of non-luminous matter, called the dark matter (DM), and is about five times more abundant than baryonic matter (Ade et al., 2014). While DM was initially controversial, it is now a widely accepted part of standard cosmology due to observations of the anisotropies in the cosmic microwave background, galaxy cluster velocity dispersions, large-scale structure distributions, gravitational lensing studies, and X-ray measurements from galaxy clusters.

Another unresolved problem in cosmology is that the detailed measurements of the mass density of the universe revealed a value that was $30 \%$ that of the critical density. Since the universe is very nearly spatially flat, as is indicated by measurements of the cosmic microwave background, about $70 \%$ of the energy density of the universe was left unaccounted for. This mystery now appears to be connected to the observation of the non-linear accelerated expansion of the universe deduced from independent measurements of Type Ia supernovae (Riess et al., 1998; Perlmutter et al., 1999; Peebles and Ratra, 2003; Sivaram, 2009).

Generally one would expect the rate of expansion to slow down, as once the universe started expanding, the combined gravity of all its constituents should pull it back, i.e. decelerate it (like a stone thrown upwards). So the deceleration parameter ( $q_{0}$ ) was expected to be a positive value. A negative $q_{0}$ would imply an accelerating universe, with repulsive gravity and negative pressure. And the measurements of Type Ia supernovae have revealed just that. This accelerated expansion is attributed to the so-called dark energy (DE).
There are several experiments to detect postulated DM particles running for many years that have yielded no positive results so far. Only lower and lower limits for their masses are set with these experiments so far. The motto seems to be 'absence of evidence is not evidence of absence'. But if future experiments still do not give any clue about the existence of DM, one may have to consider looking forward for alternate theories (Sivaram, 1994a; 1999).The best example of this is that of the orbit and position of Vulcan, which was theoretically inferred from the observation of Mercury orbit (Hsu and Fine, 2005). The deviation of its orbit, as predicted by Newtonian gravity, was attributed to the missing planet (DM). But the resolution of this discrepancy came through the modification of Newtonian gravity by Einstein and not by DM. This is unlike in the case of Uranus were the prediction and discovery were successful using DM (Neptune) theory (Kollerstrom, 2001).The original Big Bang theory had to be modified, what occurred at the beginning of the eighties, in order to solve. The Big Bang modified: Inflation .several very serious discrepancies it had accumulated when comparing it with the most accurate astronomical observations of the cosmos, specifically, concerning what happened during the very first second in the history of the Universe. It was realized that the expansion during this first second could by no means be an ordinary one, understanding by this the one that has taken place later in its evolution, say, kind of a linear one. A very special stage had to be devised to account for what occurred in this initial instant of time. The name inflation comes from the fact that the Universe expansion had to be enormous, incredibly big during an extremely small instant of time (of the order of 10-33seconds). In this infinitesimal fraction of a second the Universe expanded from the size of a peanut to that of the present Milky Way (in volume, an increase of at least 75 orders of magnitude). Actually, in the 


\section{Yinternational Research Jourma}

p-ISSN 2202-2821 e-ISSN 1839-6518 (Australian ISSN Agency)

inflationary theory the Universe begins incredibly small, some $10-24 \mathrm{~cm}$, a hundred billion times smaller than a proton. And, at the same time, during inflation it cools down abruptly (super cooling) by 5 orders of magnitude, from some $1027 \mathrm{~K}$ to $1022 \mathrm{~K}$. This relatively low temperature is maintained during the inflationary phase. When inflation ends the temperature returns to the pre-inflationary temperature; this is called reheating or thermalization because the large potential energy of the inflation field decays into particles and electromagnetic radiation, which fills the universe, starting in this way the radiation dominated phase of the Universe. Expanding universe leads to for the big bang universe However, the presence of dark energy and dark matter in the big bang universe also yields. The accelerating expansion of the expanding universe as well as the present acceleration of the big bang universe are both described by the cosmological constant.

The late-time accelerating expansion of the big bang universe is attributed to dark energy. The nature of dark energy remains elusive. Experiments to identify the quantum mechanical properties of dark matter are also ongoing. This paper has made classical propositions about the nature of dark energy and dark matter, both of which can be related to the accelerating expansion of the universe. Since the acceleration of the big bang universe and the inflationary universe are both described by the cosmological constant, it may be possible to trace the origins of dark energy and dark matter in the big bang universe to the contents of the Expanding universe.

\section{Expansion And Dark Energy}

More is unknown than is known. We know how much dark energy there is because we know how it affects the universe's expansion. Other than that, it is a complete mystery. But it is an important mystery. It turns out that roughly $68 \%$ of the universe is dark energy. Dark matter makes up about $27 \%$. The rest - everything on Earth, everything ever observed with all of our instruments, all normal matter - adds up to less than $5 \%$ of the universe. Come to think of it, maybe it shouldn't be called "normal" matter at all, since it is such a small fraction of the universe. In earlier papers "Dark Energy" and "The Nature of Dark Energy and Dark matter, it has been proposed that the accelerating expansion of the universe is due to the cosmologicalization of the principle of equivalence.

The big bang universe was preceded by the inflationary universe. The accelerating expansion of the inflationary universe was driven by the false vacuum. The false vacuum contains virtual particles. Inflation ends with the decay of the false vacuum, whereby real particles are produced and thermalized, and which initially would consist of neutrinos, and subsequently with the electroweak symmetry breaking
Vol. 10 No. 022020

82801002202004

other particles would also emerge, and all of which constitute the visible matter in the universe. As proposed, the inertial mass of the visible matter in the universe is involved in the cosmologicalization of the principle of equivalence - which is responsible for the late-time accelerating expansion of the big bang universe.

\section{Dark Matter}

It is inertial mass objects which are in cosmological freefall, because of the cosmological weak principle of equivalence. The weak principle of equivalence conveys the universality of freefall, manifesting the equivalence of gravitational and inertial mass. The universality of freefall can also be demonstrated by comparing the gravitational mass of an object in freefall with the inertial mass of a stationary object, which when observed with respect to an accelerating frame of reference gives the impression that the inertial mass of the object is also in freefall. Now in the cosmological weak principle of equivalence, instead of the impression of freefall of a stationary inertial mass object with respect to an accelerating frame of reference, we have an object with inertial mass on the surface of an expanding spherical distribution of matter, and which is in cosmological freefall in space time accelerating frame of reference, and whose acceleration is given by the cosmological constant, as in. It is objects with inertial mass, which are not directly detectable, that are proposed be the dark matter in the universe. In the accelerating expansion occurs due to the presence of the false vacuum, which consists of virtual particles. Thus, classically, there is no identifiable presence of inertial mass objects in the universe.

During expansion the scale factor expanded exponentially: where $\mathrm{H}$ is Hubble parameter. Because cosmological acceleration of inflation is roughly exponential, in one efolding time of $\sim 10-37 \mathrm{sec}$ the scale factor almost doubles, i.e., increases by almost the same amount as the previous one. In other words, in one e-folding time, the inflationary universe is expanding with almost uniform velocity. It is the series of efold expansions over e-folding times that amounts to acceleration. Thus we have a dynamically dual situation of both acceleration as well as uniform motion, depending on how we consider time: if we consider one e-folding time there is near uniform expansion, while if we consider a series of efolding times there is accelerating expansion. All the matter in the Universe exists in the form of 'normal' matter or the notoriously elusive and invisible dark matter, with the latter around six times more prolific. Curiously, scientists studying nearby galaxies in recent years have found them to contain three times less normal matter than expected, with our own Milky Way Galaxy containing less than half the expected 


\section{Yinternational Research Journal}

p-ISSN 2202-2821 e-ISSN 1839-6518 (Australian ISSN Agency)

amount "Why is it not in galaxies - or is it there, but we are just not seeing it? If it's not there, where is it? It is important we solve this puzzle, as it is one of the most uncertain parts of our models of both the early Universe and of how galaxies form." Massive and isolated spiral galaxies offer the best chance to search for missing matter. They are massive enough to heat gas to temperatures of millions of degrees so that they emit X-rays, and have largely avoided being contaminated by other material through star formation or interactions with other galaxies. Currently, there is no known physical mechanism or process underlying the phenomena attributed to dark matter and dark energy (or the finite value of $\Lambda$ if that is an accurate expression of the latter effect). This paper proposes such a physical process: a specific kind of spacetime emergence underlying a form of matter-based spacetime expansion that has not been previously taken into account. It is not itself a theory of quantum gravity. In any case, no particular theory of quantum gravity is required in order for the basic concept to be useful and applicable as a new kind of ontological understanding of the relationship between the quantum level and an emergent space time manifold. Then we discuss another aspect of the emergence process that naturally leads to the non-vanishing, but very small, value of $\Lambda$ that accounts for the "dark energy" phenomenon.

\section{The Cosmological Constant and "Dark Energy"}

The cosmological constant is the energy density of vacuum, originally introduced by Einstein (1917) as an addition to his theory of general relativity to make the universe static. Einstein abandoned the concept after Hubble's discovery that all galaxies outside the Local Group are moving away from each other. Pressure due to a cosmological constant term, $\mathrm{L}$ is given by (which is valid for any curvature):

$$
\mathrm{P}_{-\mathrm{ve}} \approx \frac{\Lambda \mathrm{c}^{4}}{8 \pi \mathrm{G}}
$$

If just $\mathrm{L}$ remains constant, matter density scales as $(1+\mathrm{Z})^{3}$. So at $\mathrm{Z} \sim 1$, matter density becomes equal to the DE density. The pressure is given by, $\mathrm{P} w \rho c^{2}, \mathrm{w}-1$ gives cosmological constant $\Lambda$.

The dark energy density is given by, $\rho \propto \rho 0 \mathrm{a}-3(1+\mathrm{W})$,

where, $\rho 0$, the initial value may be related to $\Lambda$ as $\frac{\Lambda \mathrm{c}^{4}}{8 \pi \mathrm{G}}$. The general solution for a dark energy dominated universe is:
$a(t)\left[a_{0}^{3(1+W) / 2}+\frac{3}{2}(1+W)\left(\frac{8 \pi G \rho_{0}}{3}\right)^{1 / 2} t\right]^{2 /[3(1+\mathrm{w})]}$

For w 0, we recover the matter dominated universe, $\rho \rho 0 \mathrm{a}-3$, and for $\mathrm{w} 1 / 3$, we have the radiation dominated universe: $\rho \rho$ $0 \mathrm{a}-4$.

As noted above to understand the issue of dark energy. More closely resemble the "influence network" of Knuth et al. (e.g., [20]). Nevertheless, the fact that elements of causet are added in Poissonian fashion means that the current model yields the same non-vanishing, but very tiny, value for $\Lambda$.

In natural units $(h=G=1) \Lambda$ has units of inverse length squared, and observations indicate that

$\Lambda \lesssim 1 / \mathrm{V} 1 / 2$

Based on empirical data, $\Lambda$ must be very close to zero; but to a first order approximation, one might find a very small but non-negligible values. Sorkin [22] provides such a first-order approximation, as follows. One notes (based on unimodular gravity6) that $\Lambda$ and $V$ are essentially conjugate; i.e.,

$$
\Delta \Lambda \Delta \mathrm{V} \sim 1
$$

(in natural units), analogously to the quantum mechanical uncertainty relations. Sorkin notes that this conjugate relationship between $\Lambda$ and $\mathrm{V}$ is evident from the action integral,

$$
S=-\Lambda \int(-g) 1 / 2 d 4 x=-\Lambda V
$$

Thus, if $\Lambda$ has a non-vanishing value, it may be due to its uncertainty

$$
\Delta \Lambda \sim 1 / \Delta \mathrm{V} \quad(4.6)
$$

based on any uncertainty in $\mathrm{V}$. In the causet model, $\mathrm{V}$ is proportional to the number of elements $\mathrm{N}$, since the latter specifies how many "atoms of spacetime" exist; or, in the RTI picture, how many $\mathrm{I}(\mathrm{Ei}, \mathrm{Aj})$ have been actualized. Now, given that elements are added to the (discrete) spacetime manifold in a Poissonian process, the number $\mathrm{N}$ of elements has an intrinsic uncertainty of $\mathrm{N} 1 / 2$ for any given value of the proper time $\tau$. Since $\mathrm{V}$ is a function of $\tau, \mathrm{V}$ inherits this uncertainty: $\Delta \mathrm{V} \sim \mathrm{V} 1 / 2$. If the uncertainty is the only (significant) contribution to the value of $\Lambda$, then we get precisely (1). 


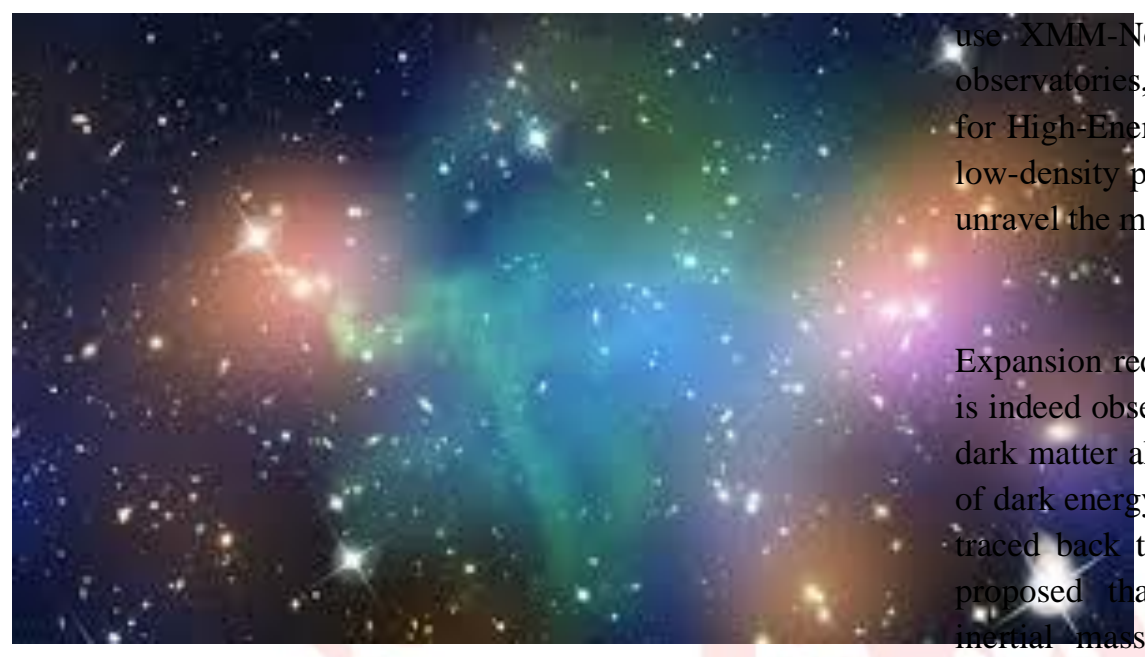

Jewton in collaboration with other high-energy , such as ESA's upcoming Advanced Telescope rgy Astrophysics, Athena, to probe the extended, parts of a galaxy's outer edges, as we continue to ystery of the Universe's missing matter."

\section{Conclusion} particles of the false vacuum, which drives the accelerating

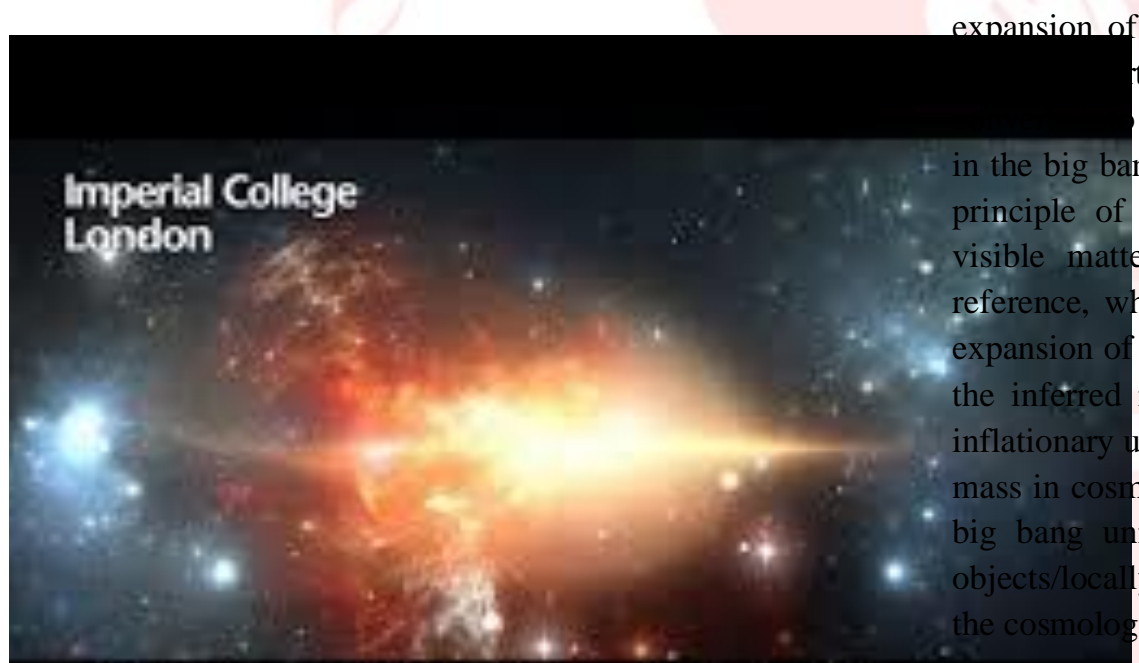

$\rightarrow$ Dark Energy : Dark Matter : Dark Gravity tial mass of a portion of the virtual particles inertial mass of real particles, i.e., visible matter ng universe. The proposition of the cosmological equivalence requires that the inertial mass of er be in a space time accelerating frame of hich is responsible for the late-time accelerating the universe. It is also proposed that a portion of inertial mass is in cosmological freefall in the iniverse. When inflation ends, the inferred inertial hological freefall converts into dark matter in the iverse, which is proposed to be inertial mass y inertial objects in cosmological freefall, due to ical weak principle of equivalence.

\section{References}

ombelli L, Henson J, Sorkin RD. Discreteness ithout symmetry breaking: a theorem. lodPhysLett. (2006) A24:2579-87.doi:

\section{Still Missing}

Results showed that the halo surrounding galaxies like the ones observed cannot contain all of the missing matter after all. Despite extrapolating out to almost 30 times the radius of the Milky Way, nearly three-quarters of the expected material was still missing. There are two main alternative theories as to where it could be: either it is stored in another gas phase that is poorly observed - perhaps either a hotter and more tenuous phase or a cooler and denser one - or within a patch of space that is not covered by our current observations or emits X-rays too faintly to be detected. Either way, since the galaxies do not contain enough missing matter they may have ejected it out into space, perhaps driven by injections of energy from exploding stars or by supermassive black holes. "In the future, scientists can add even more galaxies to our study samples and

\subsection{2/S0217732309031958}

[2]. Chadwick EA, Hodgkinson TF, McDonald GS. A gravitational development supporting MOND. Phys

Rev.(2013)D88:024036.doi:10.1103/PhysRevD.88. 024036

[3]. Cramer J. The transactional interpretation of quantum mechanics. Rev Mod Phys. (1986) 58:647-88.

[4]. Einstein A. Kosmologische Betrachtungen zur allgemeinen Relativitaetstheorie. In: Sitzungsberichte der Königlich Preussischen Akademie der Wissenschaften Part 1. Berlin. (1917). p. 142-52. 
[5]. Genzel R, Schreiber NM, Übler H, Lang P, Naab $\mathrm{T}$, Bender R. Strongly baryon-dominated disk galaxies at the peak of galaxy formation ten billion years ago.Nature (2017) 543:397-401. doi: 10.1038 /nature21685

[6]. Guth A (1981) Inflationary Universe: A Possible Solution to the Horizon and Flatness Problem. Physical Review D 23(2), 347-356.

[7]. Heisenberg W. Physics and Philosophy. New York, NY: Harper-Row (1958); https://arxiv.org/abs/1604.08112 https://dx.doi.org/10.1103/PhysRevD.23.347. https://dx.doi.org/10.5923/j.astronomy.20140301.0 2.

[8]. Huterer D, Turner MS. Prospects for probing the dark energy via supernova distance measurements. Phys Rev D (1999) 60: 1-5.

[9]. Kalita R (2014) The Nature of Dark Energy and Dark Matter. International Journal of Astronomy 3(18), 18-21.

[10]. Kalita R (2015) Dark Energy. Journal of Modern Physics 6,1007-1011. https://dx.doi.org/10.4236/jmp.2015.67105.

[11]. Kastner RE, Cramer JG. Quantifying Absorption in the Transactional Interpretation (2018). Available online at: https://arxiv.org/abs/1711.

[12]. Kastner RE. The emergence of spacetime: transactions and causal sets. In: Licata I. editor. Beyond Peaceful Coexistence. Singapore: World Scientific (2016). p. arXiv:1411.2072.

[13]. Kastner RE. The possibilist transactional interpretation and relativity. Found Phys. (2012) 42:1094-113. doi: 10.1007/s10701-012-9658-4

[14]. Kastner RE. The relativistic transactional interpretation: immune to the maudlin challenge. In: Aerts D, Dalla Chiara ML, de Ronde C, Krause D editors.Probing the Meaning and Structure of Quantum Mechanics. Singapore: World Scientific (2017).

[15]. Kastner RE. The Transactional Interpretation of Quantum Mechanics: The Reality of Possibility. Cambridge: Cambridge University Press (2012).

[16]. Kauffman S. Humanity in a Creative Universe. Oxford: Oxford University Press (2016).
[17]. Knuth K, Bahreyni N. A potential foundation for emergent space-time. J Math Phys. (2014) 55:112501. doi: 10.1063/1.4899081

[18]. Linde AD (1981) A New Inflationary Universe Scenario: A Possible Solution of the Horizon, Flatness, Homogeneity, Isotropy and Primordial Monopole Problems. Physics Letters B 108B (6), 389-393.http://dx.doi.org/10.1016/03702693(82)91219-9

[19]. Linde AD (1986) Eternally Existing SelfReproducing Chaotic Inflationary Universe. Physics Letters B175, 395-400. http://dx.doi.org/10.1016/0370-2693(86)90611-8.

[20]. Maudlin T. Quantum Nonlocality and Relativity.3rd ed. Oxford: Blackwell (2011).

[21]. Milgrom M. A modification of the Newtonian dynamics as a possible alternative to the hidden mass hypothesis. Astrophys J. (1983) 270:365.

[22]. Ng J, van Dam H. A small but nonzero cosmological constant. Int J Mod Phys.(2001) D10:49-56. doi: 10.1142/S0218271801000627

[23]. Panov A. Inverse Quantum Zeno Effect in Quantum Oscillations. (2001). Available online at: http://cds.cern.ch/record/515461/files/0108130.pdf

[24]. Rideout DP, Sorkin RD. A classical sequential growth model for causal sets.Phys Rev. (2000) D61:024002. doi: 10.1103/PhysRevD.61.024002

[25]. Rubin V, Thonnard N, Ford WK Jr. Rotational properties of $21 \mathrm{Sc}$ galaxies with a large range of luminosities and radii from NGC $4605(\mathrm{R}=4 \mathrm{kpc})$ to UGC $2885(\mathrm{R}=122 \mathrm{kpc})$. Astrophys J. (1980) 238:471-87.

[26]. Sorkin RD. Is the cosmological "constant" a nonlocal quantum residue of discreteness of the causal set type? AIP Conf. Proc. (2007) 957:14253. doi: 10.1063/1.2823750

[27]. Walsh JL, Knuth KH. An information physics derivation of equations of geodesic form from the influence network. In: MaxEnt 2015 Conference, Bayesian Inference and Maximum Entropy Methods in Science and Engineering, Potsdam NY (2015). 